\title{
Philosophiques
}

\section{Les objets de la logique classique peuvent-ils être des énoncés?}

\section{Éric Audureau}

Volume 27, numéro 2, automne 2000

URI : https://id.erudit.org/iderudit/004894ar

DOI : https://doi.org/10.7202/004894ar

Aller au sommaire du numéro

\section{Éditeur(s)}

Société de philosophie du Québec

\section{ISSN}

0316-2923 (imprimé)

1492-1391 (numérique)

Découvrir la revue

\section{Citer cet article}

Audureau, É. (2000). Les objets de la logique classique peuvent-ils être des énoncés ? Philosophiques, 27(2), 263-285. https://doi.org/10.7202/004894ar

\section{Résumé de l'article}

La philosophie de Quine n'aurait pas lieu d'être s'il y avait des propositions. L'existence de celles-ci rangerait la logique aux côtés des mathématiques ; la rupture entre, d'une part, la science et, d'autre part, le langage et le sens commun serait alors établie et le programme empiriste devrait renoncer à rendre compte du fait de la science; ce qui était précisément son but initial. Quine a si brillamment analysé les difficultés de la notion de proposition, qu'on a peu songé à examiner la nature de ce par quoi il les remplace : les énoncés. En s'appuyant sur des considérations élémentaires ressortant à des matières scientifiques philosophiquement neutres, on tente de montrer que la notion d'énoncé n'est :

(1)ni logiquement distincte (où " logiquement » signifie conforme aux lois de la logique et non pas à un mode de raisonnement particulier) de la notion de proposition : c'est une notion confuse ;

(2)ni linguistiquement claire (où « linguistiquement » signifie conforme à la théorie générale des langages) : c'est une notion obscure. 


\title{
Les objets de la logique classique peuvent-ils être des énoncés ?
}

\author{
ERIC AUDUREAU \\ CNRS ESA 6059-CEPERC Université de Provence \\ eric.audureau@wanadoo.fr
}

RÉSUMÉ. - La philosophie de Quine n'aurait pas lieu d'être s'il y avait des propositions. L'existence de celles-ci rangerait la logique aux côtés des mathématiques ; la rupture entre, d'une part, la science et, d'autre part, le langage et le sens commun serait alors établie et le programme empiriste devrait renoncer à rendre compte du fait de la science ; ce qui était précisément son but initial. Quine a si brillamment analysé les difficultés de la notion de proposition, qu'on a peu songé à examiner la nature de ce par quoi il les remplace : les énoncés. En s'appuyant sur des considérations élémentaires ressortant à des matières scientifiques philosophiquement neutres, on tente de montrer que la notion d'énoncé n'est:

(1)ni logiquement distincte (où « logiquement » signifie conforme aux lois de la logique et non pas à un mode de raisonnement particulier) de la notion de proposition : c'est une notion confuse ;

(2) ni linguistiquement claire (où « linguistiquement » signifie conforme à la théorie générale des langages) : c'est une notion obscure.

ABSTRACT. - There would be no room for Quine's philosophy if there were propositions. In that case, logic, together with mathematics, would be cut from language and common sense knowledge so that the empiricist program would not be able to account for the fact of science, whereas it was its initial aim. Quine's criticism of the notion of proposition is so bright that one often forgets to scutinize what is substituted to propositions: sentences. This paper, relying on elementary scientific considerations belonging to philosopically neutral fields of science, attempts to show that the notion of sentence is:

(1)neither logically distinct from the notion of proposition (where "logically" means in accordance with the general laws of logic): it is a confused notion,

(2)nor linguistically clear (where "linguistically" means in accordance with the principles of formal language theory): it is an obscure notion.

\section{Introduction}

1.1. Discordance de la philosophie de la connaissance et de la logique mathématique.

On constate aujourd'hui des contrastes saisissants entre les états actuels de la philosophie de la connaissance et de la logique mathématique. Un des aspects les plus frappants de cette situation est sans doute l'attachement des

PHILOSOPHIQUES 27/2 - Automne 2000, p. 263-285 
philosophes à la thèse de l'unicité de la logique, thèse selon laquelle les lois que nous devons suivre pour raisonner correctement sont uniques.

Ce contraste rappelle des précédents regrettables. Par exemple celui de Lotze qui, confronté au développement des géométries non euclidiennes, persistait à ne vouloir reconnaître qu'une forme possible à l'étendue contre les leçons qu'imposaient les progrès de la géométrie et la théorie des groupes de transformations. Ou encore la résistance de Bergson qui, face aux conclusions de la théorie de la relativité restreinte, refusait de renoncer à l'idée de simultanéité absolue.

Cette situation embarrassante, qui présente tous les aspects d'un combat d'arrière-garde, n'a évidemment pas échappé aux philosophes. Aussi justifient-ils généralement leur position inconfortable par la nécessité de préserver la logique de toute compromission avec l'ontologie, car reconnâ̂tre l'existence de plusieurs logiques revient à reconnaître plusieurs types légitimes de raisonnements, et reconnaître une telle diversité, c'est admettre que les lois logiques varient selon les choses auxquelles on les applique.

L'inconvénient de cette position doctrinale sur l'unicité de la logique est qu'elle peut conduire à remplacer les difficultés apparentes du pluralisme logique, telle que l'absence d'une définition univoque de la vérité, par des difficultés réelles portant sur les rapports de la théorie de la connaissance et de la logique. Parmi ces difficultés réelles, celle qui semble avoir suscité le moins d'incrédulité est la suivante : il y aurait un système logique qui a) possèderait les règles et les constantes de la logique classique et $b$ ) dont les choses juxtaposées aux constantes logiques (c'est-à-dire les objets désignés, par exemple, par les symboles « $A$ » et « $B$ » dans « $A \vee B$ ») seraient des énoncés, c'est-àdire des entités linguistiques.

Des considérations liminaires de logique mathématique permettent de constater l'incorrection de cette façon de voir. La popularité de cette conception, le fait que sa critique ne requiert qu'un minimum d'observations techniques et, comme on le verra, la nature de ces observations, qui relève de la syntaxe générale des démonstrations, recommande qu'on l'examine avant toute chose si on souhaite s'engager sur la voie de la réconciliation de la logique mathématique et de la théorie de la connaissance.

\subsection{Utilité publique de la question ici examinée.}

Le propos principal des réflexions qui suivent étant de montrer que les énoncés, tels que Quine les conçoit, sont des chimères, il n'exige pas, en toute rigueur, que soient examinés les motifs qui ont conduit les philosophes à les considérer comme les objets légitimes de la logique. On pourrait se borner à montrer, à partir de la seule définition des énoncés, que celle-ci ne s'accorde pas avec les propriétés formelles de la logique classique. Cependant, dans les circonstances actuelles, où la philosophie connaît une telle crise, il paraît préférable, lorsqu'on se livre à l'analyse des concepts fondamentaux de la logi- 
que, de ne pas perdre de vue les motifs et, pour tout dire, l'utilité d'une telle entreprise. L'ambition ici poursuivie, à travers la critique des énoncés, est de freiner les élans généreux du nominalisme qui dépasse trop souvent ses limites. Une remarque de J.Vuillemin illustre, pour une part, ce qu'on doit entendre, en l'espèce, par "dépasser ses limites ». Le nominalisme imprègne à un point tel la philosophie contemporaine, observe Vuillemin, que même les interprètes de Platon paraphrasent une expression comme "l'humilité (est) une vertu " par l'expression " tous ceux qui sont humbles sont vertueux". De sorte que « [1]a paraphrase porte sur les individus concrets humbles et vertueux. On a éliminé le mot abstrait humilité. À un énoncé de forme élémentaire, on a substitué un énoncé catégorique, donc complexe (" quel que soit $\mathrm{x}$, si $\mathrm{x}$ est humble, $\mathrm{x}$ est vertueux $»)$. ${ }^{1}$

Tant que ces confus débordements demeurent circonscrits aux débats philosophiques, et vu l'indifférence que la science (la logique mathématique) leur témoigne, on peut penser qu'il n'y a pas là de quoi fouetter un chat. Malheureusement, ils ne sont pas confinés aux discussions philosophiques et ils ont fait une percée insidieuse dans le domaine de l'enseignement de la logique élémentaire. Or, vu la parcimonie avec laquelle celui-ci est dispensé au cours des études universitaires de philosophie ${ }^{2}$, il est particulièrement regrettable qu'on y mêle des éléments propres à une doctrine philosophique particulière et sans laisser, qui plus est, à l'étudiant la possibilité d'entrevoir que tel est le cas. L'utilité publique des remarques qui vont suivre, si elles sont fondées, consistera donc à rappeler à ceux qui ont la charge d'enseigner les rudiments de la logique de ne pas accorder une place indue à la notion discutable d'énoncé. La fonction propédeutique de la logique, dans le cadre de l'enseignement de la philosophie, consistant principalement, d'une part, à exercer l'étudiant à la rigueur dans le raisonnement et, d'autre part, à lui donner un instrument d'analyse des systèmes philosophiques, y mêler des éléments doctrinaux revient à la dévoyer de ces objectifs.

Quine est le principal promoteur de la notion d'énoncé au détriment de celle de proposition. Comme, par ailleurs, le point de départ de toute l'œuvre de Quine était un projet pédagogique ${ }^{3}$, qu'à ce projet est venu s'associer une

1. Vuillemin, Jules, «Les formes fondamentales de la prédication: un essai de classification ", Cahiers du DREUG 4, 1984, pp.9-30. p.12 pour la citation.

2. En écrivant ces mots, c'est, bien entendu, à l'université française qu'on pense. Cette observation ne doit pas être comprise comme un plaidoyer pour le développement de l'enseignement de la logique. La connaissance des rudiments de la logique mathématique est indispensable à la compréhension de la philosophie contemporaine, mais la connaissance de rudiments de mathématiques est tout aussi indispensable à la compréhension de la philosophie ancienne, tout comme celle d'un minimum de physique l'est pour l'intelligibilité de la philosophie classique. C'est donc plutôt vers un enseignement élémentaire de ces trois sciences qu'il faudrait s'orienter.

3. Faire comprendre à des étudiants « undergraduate » ce qu'est un procédé complet de démonstration pour le calcul des prédicats et les rendre capable de transcrire dans le langage de la logique les expressions du langage ordinaire en un semestre. Hahn, L.E. et Schilpp, Paul A., dir. The Philosophy of W. V. Quine, La Salle, Open Court, 1986, p.644. 
orientation philosophique ${ }^{4}$ et que ces deux motifs sont devenus indissociables dans l'élaboration de ses idées maîtresses, telles que son renoncement aux propositions et aux propriétés au profit des énoncés et des classes ${ }^{5}$, on rappelera rapidement les grands traits de cette doctrine.

\subsection{Rappel sommaire de la doctrine de Quine.}

Les mathématiques, et donc la théorie des ensembles, ne peuvent être ontologiquement neutres puisqu'elles nous engagent à quantifier sur des classes, et donc à postuler leur existence. La logique, de par sa place au sein du tissu de nos connaissances, doit être ontologiquement neutre puisqu'elle est la garante de l'invariance des conditions sous lesquelles la communication est possible.

Les énoncés constituent une classe distinguée de formes linguistiques. Leur trait distinctif, ce qui les oppose aux autres formes linguistiques, est qu'ils peuvent être vrais ou faux ${ }^{6}$. Aux énoncés peuvent être appliqués des fonctions telles que non ou et. La fonction non prend un énoncé $p$ comme argument et donne l'objet non(p). Non $p$ est toujours un énoncé, car non transforme la valeur de vérité (le vrai ou le faux) d'un énoncé en la valeur opposée. La forme linguistique donnée, " $\mathrm{p}$ ", qui était un énoncé $p$, est bien transformée, par la préfixation de «non ", en un énoncé (puisque «non p » a une valeur de vérité, celle de non $(p))$. Les expressions auxquelles on peut associer une fonction de vérité doivent être distinguées de tous les autres éléments du lexique. On peut les dire universelles, au sens où elles valent pour toutes les langues ${ }^{7}$. De ce fait, les fonctions de vérité sont les seules règles du parler à échapper à l'indétermination de la traduction, ce qui les rend garantes de la possibilité de la communication. Cette propriété écarte l'éventualité d'une mentalité pré-logique ${ }^{8}$, tout comme elle exclut la considération de systèmes logiques autres que la logique classique. En effet la négation classique (la fonction non) appliquée deux fois à un énoncé $(\operatorname{non}(\operatorname{non}(p)))$ laisse sa valeur de vérité inchangée. Tandis que la négation intuitioniste, par exemple, appliquée dans les mêmes conditions, laisse la valeur de vérité de l'expression résultante indéfinie.

L'adoption de la notion d'énoncé est liée à un certain nombre de thèses : l'indétermination de la traduction et ses limites, la neutralité ontologique, l'unicité, et, enfin, le "classicisme " de la logique, qui sont toutes essentielles à la philosophie de Quine. Il reste à comprendre pourquoi les propositions ne peu-

4. Dans la conception de la théorie de la concaténation comme syntaxe générale (exposée, par exemple, dans Quine, William V.O., "Concatenation as number theory " in Selected Logic Papers, New York, Random House, 1966) ibid p.645

5. Mais également au profit de l'insistance sur la distinction entre implication et conditionnel et, plus généralement sur la distinction entre mention et usage.

6. Quine, William V.O., Méthodes de logique, p.19, Paris, A.Colin, 1973, p.19.

7. Plus exactement les mots logiques sont universels par provision. Quine considérant qu'on ne peut exclure qu'une découverte scientifique future puisse nous amener à réviser les lois de la logique, puisque celles-ci sont solidaires de la totalité de nos connaissances.

8. Quine, William V.O., Word and Object, Cambridge, MIT Press, 1960, p.58. 
vent remplir un rôle similaire à celui des énoncés, puisque, généralement, on est enclin à penser que ce sont là les êtres dont on peut dire qu'ils sont vrais ou faux. Une proposition est la classe des énoncés ayant la même signification. Mais la relation d'identité de signification " est dénuée de sens objectif au niveau des énoncés ${ }^{9}$, car l'identité de signification dépend de la synonymie qui, à son tour dépend de l'identité de signification. Cette circularité explique les difficultés de la notion de signification, qui devient incompréhensible lorsqu'on tente de procéder à des substitutions d'énoncés identiques par la signification. Telle est, sommairement résumée, la position de Quine.

Cependant, l'impossibilité d'établir une correspondance ferme entre énoncés et propositions ne permet de disqualifier la notion de proposition qu'à partir de l'instant où la notion d'énoncé est elle-même assurée. Pour vérifier que cette condition n'est pas remplie, on ne préjugera pas de la nature des objets manipulés par la logique mathématique. On se bornera à observer que celleci manipule des formules. De même on ne préjugera pas de la nature de la logique. On se limitera à constater que, aux côtés de la logique classique, l'existence de systèmes de logiques " déviants » est historiquement attestée et on se demandera plutôt si la logique mathématique met à notre disposition un mode de représentation laissant apparaittre les propriétés des logiques «non classiques » au même titre que celles des logiques classiques, sans privilégier, dans la formalisation, les traits caractéristiques d'un système au détriment de ceux d'un autre système. Or une telle théorie de la représentation existe, c'est le calcul des séquents de Gentzen. L'examen des propriétés générales de ce calcul nous permettra de constater :

1) qu'il est philosophiquement neutre,

2) qu'il permet de vérifier la véracité de la thèse fondamentale, due à Quine, de l'indétermination de la traduction : celle-ci vaut pour les langages logiques,

3) que de 1) et de 2), il suit que logique classique et logique intuitioniste sont égales en droit quant à la clarté de leurs principes ${ }^{10}$,

4) que la logique, classique, intuitioniste ou autre, ne peut jamais être dite neutre, selon une notion de neutralité plus restrictive que celle retenue par Quine lorsqu'il parle de "neutralité ontologique »,

5) qu'il en découle que la notion d'énoncé est confuse, c'est-à-dire indistinguible de celle de proposition, et obscure, car elle est le produit d'un mélange de considérations linguistiques et logiques.

Examinons le calcul des séquents.

9. «S'il y avait des propositions, elles induiraient une certaine relation de synonymie ou d'équivalence entre les énoncés eux-mêmes : les énoncés qui expriment la même proposition seraient équivalents. Or [...] la relation d'équivalence appropriée est dénuée de sens objectif au niveau des énoncés. » Philosophy of Logic, Engelwood Cliffs, Prentice-Hall, 1970, p.3. Trad. française :Philosophie de la logique, Paris, Aubier, 1975, p.11. Sauf mention expresse, les références à cet ouvrage revoient à la traduction française.

10. Ici la thèse de l'indétermination se retourne contre Quine. 


\section{Rappels sur le calcul des séquents et examen de certaines de ses propriétés}

La doctrine selon laquelle il ne peut exister qu'une logique, qu'il s'agisse de celle de Quine ou d'une autre, revient à soutenir ceci : aux mots du langage usuel «ou », " et », " non », etc...il faut associer un sens univoque. Par exemple que l'on dise "mes clés sont dans mon pantalon ou dans mon veston » ou «quelque soit $\mathrm{x} \mathrm{P}(\mathrm{x})$ ou non $\mathrm{P}(\mathrm{x})$ », les emplois de « ou » n'auront pas à être différenciés. Chez Quine la nature immanente des constantes logiques, nature à laquelle elles doivent leur signification unique, relève d'une sorte de miracle comme le montre l'impossibilité de passer de façon univoque de la logique du verdict à la logique des fonctions de vérité. ${ }^{11}$ Or à la révélation il vaut mieux préférer la logique car, «si la logique à l'état pur n'est pas impérative, qu'est-ce qui l'est ? "12

\subsection{Description informelle du calcul des séquents. ${ }^{13}$}

Un séquent est une expression de la forme $A_{1}, \ldots, A_{n} \vdash B_{1}, \ldots, B_{m}$ où les $A_{i}$ et les $B_{j}$ sont des expressions bien formées d'un langage logique au sens habituel. La signification informelle de l'expression précédente est la suivante : « $B_{1}$ ou...ou $B_{m}$ est déduite de $A_{1}$ et...et $A_{n}$ ». Le dictionnaire pour passer de l'expression formelle à la signification informelle est donc le suivant : « $\vdash$ » signifie " déduire » et la virgule ", » signifie " et » ou " ou » suivant qu'elle est respectivement à gauche ou à droite de $\vdash$. On peut estimer, d'après la signification informelle de l'expression formelle, que celle-ci entretiendra un certain rapport avec la formule de logique $A_{1} \ldots A_{n} \vdash B_{1} \vee \ldots \vee B_{m}$. On voit donc déjà qu'un des buts du calcul des séquents est de permettre d'analyser les rapports qui unissent l'expression formelle à sa signification informelle. Lorsque, faisant du calcul ou de la déduction logique, nous employons les signes $\wedge, \vee, \neg$, etc...nous les tenons pour les représentants légitimes des mots du langage usuel « et », " ou », " non », etc. parce que, dans les apparences, tout y invite. Mais, puisque le propre de la science est de nous révéler ce qu'il peut y avoir de trompeur dans les apparences, on peut s'attendre à ce que la

11. Cf. Le $\mathbb{S} 20$ de The Roots of Reference, Open Court, La Salle, 1973. L'examen détaillé de cette question dépasse les limites de cet article.

12. Philosophie de la logique, p.120.

13. Le rôle prêté ici au calcul des séquents est inspiré des réflexions de Scott, Dana, "On engendering an illusion of understanding”, Jour. of Philosophy, 1971, pp.787-807. À savoir que ce calcul doit être avant tout considéré comme un cadre de représentation des "principes les plus généraux de l'inférence déductive » (p.793), plutôt que comme un outil de la théorie de la démonstration. Scott montre comment les opérateurs modaux de la logique aléthique peuvent être analysés grâce au calcul des séquents. Rappelons que cet essai de Scott est à l'origine d'une série de travaux dans lequel le calcul des séquents sert de cadre de représentation pour classer les systèmes logiques ou pour analyser les mots logiques. Voir par exemple Dosen, Kosta, "Logical constants as punctuation marks", Notre-Dame Jour. of Formal Logic, Vol.30, n³, 1989, et les références données dans cet article. 
logique mathématique, qui est une science, nous révèle des propriétés inattendues sur la nature des constantes logiques et, en particulier, que celles-ci ne correspondent pas au sens que nous attribuons spontanément aux mots logiques auxquels elles sont associées à travers l'usage du langage ordinaire.

On fait du calcul sur les séquents à l'aide de règles qui doivent être divisées en trois groupes $(\mathrm{X}, \mathrm{Y}, \mathrm{Z}, \ldots$ désignent respectivement des expressions de la forme $A_{1}, \ldots, A_{m}, B_{1}, \ldots, B_{n}, C_{1}, \ldots, C_{p}, \ldots, c^{\prime}$ 'est-à-dire des suites d'expressions bien formées) ${ }^{14}$ :

1) Les règles de l'identité

\begin{tabular}{lc}
\hline axiome d'identité & $A \vdash A$ \\
coupure & $\frac{X \vdash Y, A \quad A, Z \vdash U}{X, Z \vdash Y, U}$ \\
\hline
\end{tabular}

2) Les règles de données'5

\begin{tabular}{lll}
\hline \multirow{2}{*}{ affaiblissement } & \multicolumn{1}{c}{ à gauche } & à droite \\
\multirow{2}{*}{ contraction } & $\frac{X \vdash Y}{X, A \vdash Y}$ & $\frac{X \vdash Y}{X \vdash Y, B}$ \\
expansion & $\frac{X, A, A \vdash Y}{X, A \vdash Y}$ & $\frac{X \vdash Y, A, A}{X \vdash Y, A}$ \\
échange & $\frac{X, A \vdash Y}{X, A, A \vdash Y}$ & $\frac{X \vdash Y, A}{X \vdash Y, A, A}$ \\
& $\frac{X, A, B, Z \vdash Y}{X, B, A, Z \vdash Y}$ & $\frac{X \vdash Y, A, B, Z}{X \vdash Y, B, A, Z}$ \\
\hline
\end{tabular}

14. Cette présentation des règles du calcul des séquents est simplifiée, puisque, en particulier, les règles pour l'introduction des quantificateurs n'y sont pas données. Telle quelle, elle suffit à l'exposé de notre propos.

15. On appelle habituellement règles structurales ce qui est ici appelé règles de données. En empruntant cette modification terminologique à Crocco, Gabriella, Les fondements logiques $d u$ raisonnement contextuel, Padoue, Unipress, 1996, p.13, on ne se borne pas à reconnaître que les règles logiques sont également structurales, mais on dispose également d'un terme commode pour désigner les choses sur lesquelles portent les règles du calcul des séquents : données. 
270 Philosophiques / Automne 2000

3) Les règles logiques

\begin{tabular}{lcc}
\hline \multirow{2}{*}{ conjonction } & $\frac{X, A \vdash Y}{X, A \wedge B \vdash Y}$ & $\frac{X \vdash A, Y \quad X \vdash B, Y}{X \vdash A \wedge B, Y}$ \\
\multirow{2}{*}{ disjonction } & $\frac{X, A \vdash Y \quad X, B \vdash Y}{X, A \vee B \vdash Y}$ & $\frac{X \vdash A, Y}{X \vdash A \vee B, Y}$ \\
\multirow{2}{*}{ négation } & $\frac{X \vdash Y, A}{X, \neg A \vdash Y}$ & $\frac{X, A \vdash Y}{X \vdash \neg A, Y}$ \\
conditionnel & $\frac{X \vdash Y, A \quad Z, B \vdash W}{X, A \rightarrow B, Z \vdash Y, W}$ & $\frac{X, A \vdash Y, B}{X \vdash Y, A \rightarrow B}$ \\
\hline
\end{tabular}

Considérons d'abord les règles logiques. Elles gouvernent le comportement des constantes logiques en rendant explicites, en termes de séquents, les conditions sous lesquelles on peut les introduire. Ces règles sont symétriques au sens où elles permettent de considérer les cas où la constante est introduite dans la partie gauche ou la partie droite d'un séquent. Elles nous renseignent également sur les propriétés de $\vdash$. Les règles de la négation et du conditionnel sont telles qu'on peut se demander légitimement si elles portent sur le comportement de $\vdash$ ou de $\neg$ et $\rightarrow$. Nous y reviendrons. Elles contiennent également une autre forme de réciprocité entre $\vdash$ et les constantes logiques, cette fois $\wedge$ et $\vee$ plus particulièrement (mais également $\rightarrow$ ) puisque, tout autant qu'elles permettent d'introduire les constantes logiques, elles transforment deux séquents en un seul, ou, si l'on préfère, elles sont un procédé de suppression des signes $\vdash$.

Les règles de données transforment également les séquents comme le font les règles logiques, mais en un sens différent. Une image, hasardeuse, comme toutes les images, peut illustrer ce qui distingue les règles logiques des règles de données. Ces dernières traitent des transformations moléculaires des séquents tandis que les premières décrivent leurs transformations atomiques. Les molécules, ce sont nos expressions $\mathrm{A}, \mathrm{B}$, etc.... Les règles de la conjonction, par exemple, font surgir une nouvelle molécule de composition $A \wedge B$. La conjonction à droite est comme une transformation physico-chimique. A partir de deux éléments simples $A$ et $B$, elle donne une molécule d'un composé $A \wedge B$. Les transformations physico-chimiques consomment de l'énergie. Les transformations logiques consomment de l'information. L'information perdue dans la transformation qui donne la conjonction à droite est celle portant sur l'existence originaire de deux séquents (deux signes $\vdash$ ) car, après application de la règle, nous n'avons plus qu'un séquent. Les règles de données, comme on le constate en les regardant, n'opèrent pas de transformation à l'intérieur des formules (des molécules), pas plus qu'elles ne suppriment de $\vdash$.

Les règles d'échange modifient l'ordre des formules. Les règles de contraction permettent de réduire plusieurs occurrences d'une même formule à une seule occurrence. Les règles d'expansion jouent un rôle inverse. Les règles d'affaiblissement peuvent être distinguées des autres règles de données, 
car, contrairement à celles-ci, elles introduisent de nouvelles formules dans un séquent. L'interprétation des règles d'affaiblissement gagne à être illustrée par un exemple. Soit le séquent $\mathrm{A}, \mathrm{B} \vdash \mathrm{C}$. Il peut être informellement interprété comme : de $\mathrm{A}$ et $\mathrm{B}$ on déduit $\mathrm{C}$. $\mathrm{A}$ et $\mathrm{B}$ étant comme des hypothèses et $\mathrm{C}$, comme une conclusion de la déduction. Puisque, à partir de $\mathrm{A}$ et $\mathrm{B}$, on peut déduire $\mathrm{C}$, on peut a fortiori faire de même à partir de $\mathrm{A}, \mathrm{B}$ et $\mathrm{D}$. La déduction est affaiblie, car les hypothèses sont augmentées. De même pour l'affaiblissement à droite. Une conclusion « A ou B » est moins ferme, plus faible, qu'une conclusion unique A.

Les règles de l'identité, enfin, sont mal nommées puisqu'il y a là une règle et un axiome. Deux choses de nature différente comme nous allons bientôt le voir.

L'axiome dit que, d'une formule donnée, on peut déduire elle-même, et qu'une formule peut être déduite d'elle-même. C'est ce qu'on doit appeler l'axiome de la logique, comme l'avait vu Leibniz. Quand cet axiome n'est pas respecté on quitte la logique. On rencontre de plus en plus souvent des cas de calcul formel employant peu ou prou les règles logiques et les règles de données mais qui, par ailleurs, violent l'axiome d'identité ${ }^{16}$. La seule chose que l'on doit dire de ces calculs est qu'ils ne sont pas (que) de la logique. La prolifération des systèmes logiques fait qu'on considère parfois comme pressante la question de la démarcation entre ce qui relève de la logique et ce qui lui est étranger. L'axiome d'identité donne une condition nécessaire pour qu'un calcul appartienne à la logique. En demeurant platement au niveau formel, l'exigence exprimée par cet axiome est la suivante : une fois définie clairement, à travers des règles explicites d'assemblage de symboles, ce que sont les formules, une formule a la même valeur qu'elle-même au sens où le

16. C'est ce qui ressort de l'examen de certains systèmes employés pour formaliser la négation par échec en théorie de la programmation logique. Par exemple Kunnen, K., « Signed data dependencies in logic programs ", Jour. of Logical Programming, 6, pp.231-45, 1989, propose des conditions sur la formation des clauses des programmes logiques qui reviennent, une fois faite la traduction du langage des clauses de Horn dans celui du calcul des séquents, à exclure les séquents de la forme $\neg \mathrm{A}(\mathrm{x}) \vdash \neg \mathrm{A}(\mathrm{x})$. Bien évidemment l'axiome d'identité n'est pas rejeté en tant que tel. Plutôt, une fois définies de façon usuelle la syntaxe et la sémantique du langage, on ajoute des conditions formelles ad hoc qu'on pourrait, par analogie avec la nomenclature de la grammaire, qualifier de morphologiques, puisqu'elles conduisent à des restrictions sur la syntaxe justifiées par des conditions d'usage altérant la sémantique. L'altération sémantique en question consistant à essayer de conserver les propriétés de "ne pas ", telles qu'elles apparaissent dans une locution comme " être ou ne pas être ", dans un usage strictement restreint au cas où « ne pas » est employé dans « être dérivable ou ne pas être dérivable ». Une des leçons des difficultés de la programmation logique semble donc être qu'il n'y a pas de mesure commune d'identité dans les deux locutions " être identique ou ne pas être identique " et " être dérivablement identique et ne pas être dérivablement identique ».

Pour une discussion de certaines orientations de la philosophie de la logique (en particulier celle suivie par Tennant, Neil, Anti-realism and Logic, Londres, Clarendon Press, 1987) soulevant des difficultés pour le principe logique d'identité voir Crocco, Les Fondements logiques du raisonnement contextuel, ch. 2 . 


\section{Philosophiques / Automne 2000}

calcul logique ne doit jamais nous amener à réviser en cours de route notre définition explicite de ce qu'est une formule ${ }^{17}$. Si nous délaissons un instant ce qui découle d'un examen purement formel pour nous tourner vers des considérations historiques élémentaires nous voyons que le calcul des séquents permet de comprendre la profondeur de vue de Leibniz. Celui-ci voyait un lien analytique entre le principe de contradiction et le principe d'identité tout en attribuant une priorité logique au premier. En appliquant la négation à gauche à l'axiome d'identité on obtient $\mathrm{A}, \neg \mathrm{A} \vdash$. Soit, en signification informelle, de A et non A rien ne doit être déduit, c'est-à-dire le principe de contradiction. Comme Leibniz félicite Aristote d'avoir fait cette découverte on voit que la logique mathématique moderne s'accorde avec le point de vue des deux plus grands logiciens du passé.

La règle de coupure est parfois comptée parmi les règles de données, mais l'examen de son rôle invite à la ranger aux côtés de l'axiome d'identité puisqu'elle exprime, entre autres, la propriété suivante : si une même formule apparaît dans la partie droite d'un séquent $S_{1}$ et dans la partie gauche d'un séquent $S_{2}$, alors on peut inférer un séquent $S_{3}$ où $A$ ne figure plus et dont les parties gauche et droite contiennent respectivement les parties gauches et droites de $S_{1}$ et $S_{2}$. La règle de coupure permet donc de composer des déductions sous condition de l'identité entre une conséquence et une hypothèse de ces deux déductions. Elle exprime, sous une autre forme, une condition nécessaire, toujours liée à l'identité, pour qu'un calcul soit une logique : une relation de déduction logique est transitive. L'axiome exprime le principe d'identité, la règle de coupure donne les conditions d'usage de l'identité. Ou, comme on le dit parfois justement, l'axiome est la forme statique de l'identité, la règle de coupure sa forme dynamique, puisque cette dernière règle utilise l'identité dans le cours des démonstrations. ${ }^{18}$

\subsection{Règles et axiomes.}

La première chose à observer dans cette formulation des lois de la logique est la manière dont elle se distingue de la méthode axiomatique de Hilbert et Russell. Dans celle-ci on tient pour vraies un certain nombre de formules de base et on déduit à partir d'un nombre restreint de règles d'inférence, le plus souvent le principe de substitution uniforme et le modus ponens (et la règle de généralisation universelle dans le cas du calcul des prédicats), l'ensemble de toutes les formules vraies. Dans le cas du calcul des séquents, le recours aux formules de base disparaît au profit de la formalisation de la relation $\vdash$, qui n'est caractérisée que par des règles d'inférence.

17. Voir la note précédente..

18. G. Crocco, Fondements logiques..., p.14. Girard, Jean-Yves, et alii., Proof and Types, Cambridge, Cambridge University Press, 1989, pp. 3 et 31. 
Pour mesurer toute la portée de la différence entre la méthode axiomatique et l'approche de Gentzen, considérons l'une de ces formules de base, par exemple l'un des axiomes du calcul des propositions retenus par Hilbert et Ackermann dans leur célèbre monographie ${ }^{19}, \mathrm{~A} \rightarrow \mathrm{A} \vee \mathrm{B}$. Cette formule est élevée au rang d'axiome, car elle semble exprimer une vérité indubitable. De quel ordre est cette vérité ? Relève-t-elle d'un principe du calcul ou d'une évidence intellectuelle, c'est-à-dire d'un postulat logique auquel on ne saurait, dans aucune circonstance, renoncer ? Un peu des deux, car, d'une part, elle code ce qu'exprime distinctement la règle d'affaiblissement à droite, comme l'indique la signification informelle, et, d'autre part c'est un théorème de la logique classique comme de la logique intuitioniste. Telle est la difficulté de la méthode axiomatique en logique mathématique : ses principes mi-intuitifs, mi-abstraits confondent sous un même chef les règles et ce qu'on obtient en appliquant les règles, c'est-à-dire les choses démontrées inconditionnellement (sans hypothèses), qu'on appelle vérités logiques.

Le calcul des séquents permet toutefois de retrouver tout ce que la méthode axiomatique permet d'exprimer, mais en en relativisant la nature, et c'est là que réside la source de sa neutralité et, par là même, sa plus grande généralité. Reprenons l'exemple de l'axiome du paragraphe précédent. On vérifie rapidement que celui-ci est un théorème du calcul des séquents : $\mathrm{A} \vdash \mathrm{A}$ (axiome), $\mathrm{A} \vdash \mathrm{A} \vee \mathrm{B}(\vee$ à droite),$\vdash \mathrm{A} \rightarrow \mathrm{A} \vee \mathrm{B}(\rightarrow$ à droite). Il s'agit bien d'un théorème, le fait qu'aucun symbole n'apparaisse à gauche du $\vdash$ devant être interprété comme une démonstration inconditionnelle de ce qui est à sa droite.

On ne peut donc, comme on le croit parfois, considérer que la méthode axiomatique et le calcul des séquents ne sont que des modes différents de présentation de la logique.

\section{La neutralité philosophique du calcul des séquents}

On dit d'un point de vue qu'il est neutre lorsque, considérant plusieurs parties qui s'opposent, il s'abstient d'épouser une des thèses propres à l'une des parties. Ce n'est évidemment pas en ce sens que Quine considère que la logique est neutre. La logique est neutre dit-il, car elle ne nous engage pas à reconnaître, comme le font les mathématiques, l'existence d'entités suprasensibles. La neutralité que nous recherchons dans le calcul des séquents est préliminaire à l'examen de la neutralité ontologique, au sens donné par Quine à cette notion. Ce calcul est neutre face à l'opposition énoncé/proposition car, nous venons de le voir, il ne manipule que des formules. Ce n'est qu'au terme d'un examen plus avancé qu'on pourra décider si ces formules désignent des énoncés ou des propositions. Mais le calcul des séquents est

19. Hilbert, David et Ackermann, Wilhelm Grundzüge der theoretischen Logik, Springer 1928. 


\section{Philosophiques / Automne 2000}

neutre à un autre égard. Défendre l'idée que la logique s'occupe d'énoncés conduit de manière concomitante, dans le système philosophique de Quine, à soutenir, comme nous l'avons rapidement vu, que la logique ne peut être que la logique classique ${ }^{20}$. La neutralité, telle qu'on l'entend ici, consiste également à ne pas admettre que « les noms et les notations de la négation et de la disjonction ne se transposent à une logique déviante, telle l'intuitioniste, que par une analogie grossière et quelque peu arbitraire ${ }^{21}$ ou que les constantes de cette logique ont " une sorte de sens intuitif » 22 avant d'avoir examiné les enseignements littéraux de la logique mathématique sur la nature des constantes logiques. Rappelons donc brièvement ce qu'est l'intuitionisme, après quoi nous pourrons examiner la caractérisation logique de cette doctrine.

\subsection{Qu'est-ce que l'intuitionisme?}

Avant que Cantor et d'autres n'introduisent les ensembles transfinis dans les mathématiques, celles-ci pouvaient admettre sans réserves des procédés de démonstration indirecte, ou par l'absurde, une démonstration indirecte étant en principe convertible en une démonstration directe ou constructive. Les démonstrations par l'absurde pouvaient être considérées comme des pisaller. Plus simples que les preuves directes, ou constructives, elles sont comme un encouragement à rechercher de telles preuves. Dès lors que sont introduits les ensembles transfinis, cette possibilité de principe d'une conversion de toute preuve par l'absurde en une preuve directe disparait. Toutefois, ce n'est là que l'amorce de la crise des fondements des mathématiques. La crise proprement dite survint lorsqu'on constata que les principes généraux sur lesquels s'appuient les démonstrations indirectes irréductibles à des preuves constructives conduisaient à des paradoxes. Pour remédier à ces difficultés, un courant de pensée se fit jour, au sein de la communauté des mathématiciens, qui exigea qu'on règle l'emploi des méthodes de démonstration de sorte que les mathématiques soient exemptes de paradoxes. On appelle intuitioniste ce courant de pensée. On pense parfois que ces controverses ne concernent qu'un aspect épiphénomènal des mathématiques, qui n'intéresse que les philosophes et qui est sans incidence sur le développement des mathématiques. Plutôt que de mentionner les noms des mathématiciens, les plus grands de ce siècle, qui ont pris part à cette controverse, bornons-nous à en

20. Cette affirmation doit être nuancée, Quine admettant soit que l'évolution de nos connaissances pourrait nous conduire à changer de logique (cas de la mécanique quantique), soit que l'enracinement linguistique de l'épistémologie est compatible avec la logique intuitioniste. Mais la maxime de la mutilation minimum éliminant, pour l'heure, ces éventualités, la logique ne peut être que la logique classique. Pour Quine le pluralisme logique équivaut au relativisme, par conséquent la logique doit être unique.

21. Quine, Philosophie de la logique, p.139.

22. Ibid.,p.130. 
observer les conséquences à travers un exemple. Selon les intuitionistes, le théorème : Sur la droite réelle $\mathbf{R}$ munie de sa relation d'ordre naturel, toute partie majorée non vide a une borne supérieure et toute partie minorée non vide a une borne inférieure, qu'on enseigne en première année de licence de mathématiques, est indémontrable.

Une preuve par l'absurde a la forme générale suivante. On veut savoir si A est vraie. On suppose non A et on démontre qu'il en découle une absurdité. De non A on peut alors dire que découle non non A. Comme on considère que non non $\mathrm{A}$ est identique à $\mathrm{A}$, la démonstration est achevée. Ce que récusent les intuitionistes, c'est cette identification entre " non non » et " oui » : de l'inexistence d'une preuve de non $\mathrm{A}$ on ne peut conclure à la vérité de $\mathrm{A}$. Les réserves intuitionistes peuvent être formulées différemment : le principe selon lequel soit A est vraie soit non A l'est est inadmissible.

\subsection{Comment caractériser l'intuitionisme?}

La logique intuitioniste se distingue de la logique classique en repoussant le principe du tiers exclu. On peut déduire le tiers exclu au moyen des inférences suivantes:

$$
\frac{\frac{A \vdash A}{A \vdash A \vee \neg A}}{\frac{\vdash \neg A, A \vee \neg A}{\vdash A \vee \neg A, A \vee \neg A}} \frac{\vdash A \vee \neg A}{P A}
$$

disjonction à droite, négation à droite, disjonction à droite, contraction à droite. Pour éliminer la possibilité d'une telle déduction, trois possibilités sont offertes : modifier la règle de la disjonction, modifier la règle de la négation, supprimer la règle de contraction à droite. Du point de vue de la signification informelle d'un séquent, la règle de contraction paraît sans conséquence. Quelle différence entre déduire deux fois le tiers exclu et le déduire une fois ? À quoi on peut ajouter que les intuitionistes pensent souvent que s'il y a une difficulté dans la logique classique, c'est bien dans la nature obscure de la négation, source principale de l'usage inconditionné de la dialectique, qu'il faut la chercher. Par conséquent avant de répondre à la question " comment amender les règles du calcul des séquents de sorte que le principe du tiers exclu n'y soit plus déductible ? ", on doit constater $1^{\circ}$ ) que plusieurs possibilités nous sont offertes et $2^{\circ}$ ) que les apparences poussent à modifier les règles logiques plutôt que les règles de données. Mais le calcul des séquents est un calcul formel. C'est une méthode mathématique pour examiner objectivement les rapports entre la signification informelle et la part de cette signification véhiculée par les symboles $\neg, \vee, \ldots$ dans le langage logique. Interprété à la lettre, il proscrit donc d'identifier $\vdash \mathrm{A} \vee \neg \mathrm{A}$, 
$\mathrm{A} \vee \neg \mathrm{A}$ avec $\vdash \mathrm{A} \vee \neg \mathrm{A}$. De plus, faire porter la modification sur les règles logiques serait retomber dans les difficultés de la méthode axiomatique auxquelles le calcul des séquents se propose justement de remédier. Gentzen choisit donc de modifier les règles de données en adoptant la condition générale suivante : un séquent intuitioniste n'a au plus qu'une formule à droite. Cette condition revient à supprimer les règles structurales à droite, puisque celles-ci n'ont plus lieu d'être. Ces considérations appellent trois remarques.

$1^{\circ}$ ) La formalisation de l'intuitionisme retenue par Gentzen n'a pas pour thème explicite le tiers exclu ; le principe explicitement formalisé, ici, comme propre à l'intuitionisme, c'est l'univocité de la chose déduite. Cette caractéristique est propre aux enchaînements déductifs qui déterminent une construction. $\mathrm{X} \vdash \mathrm{A}$ ne permet pas d'inférer $\mathrm{X} \vdash \mathrm{A}$, B. La relation $\vdash$ n'a qu'une image ; rapportées à leur signification informelle, les démonstrations intuitionistes sont des fonctions.

$2^{\circ}$ ) L'ordre ici suivi pour présenter informellement les notions essentielles du calcul des séquents pourrait laisser supposer que l'on y définit la logique intuitioniste à partir de la logique classique (en privant cette dernière de quelque chose, en l'occurrence les règles de données à droite). La thèse de la neutralité du calcul des séquents en serait affectée, et ceux qui pensent que les logiques «non-classiques » sont, en dernier recours, toujours subordonnées aux principes de la logique classique y verraient de l'eau apportée à leur moulin. Cette objection doit être repoussée. Les règles logiques du calcul des séquents sont, dans l'exposé de Gentzen, définies à partir de leur signification opérationnelle tel que l'exige l'intuitionisme. On peut donc tout aussi bien dire que c'est l'adjonction de règles supplémentaires à la logique intuitioniste qui permet de définir la logique classique. Cette remarque doit être complétée par la suivante.

$3^{\circ}$ ) La présentation succincte du calcul des séquents donnée ci-dessus est incomplète. Les progrès de la formalisation des logiques "non classiques " ont révélé une distinction formelle significative entre deux types de disjonction et de conjonction que le seul examen des logiques classique et intuitioniste ne laissait pas apparaître. La règle de la disjonction à gauche donnée plus haut s'écrivait

$$
\frac{X, A \vdash Y X, B \vdash Y}{X, A \vee B \vdash Y}
$$

elle s'applique donc uniquement dans les cas où $\mathrm{A}$ et $\mathrm{B}$ sont entourées des mêmes listes de formules ( $\mathrm{X}$ et $\mathrm{Y}$ ). Or les cas où les contextes de $\mathrm{A}$ et $\mathrm{B}$ sont différents correspondent à une distinction pertinente et on doit en toute rigueur discerner la disjonction additive (ci-dessus) de la disjonction multiplicative

$$
\frac{X, A \vdash Y \quad Z, B \vdash W}{X, Z, A \vee B \vdash Y, W}
$$


et la même observation vaut pour la règle de la disjonction à droite et pour les règles de la conjonction. ${ }^{23}$ Lorsqu'un calcul logique contient toutes les règles de données, cette distinction s'évanouit. Si on considère que la logique intuitioniste est obtenue par suppression de certaines règles de données, il faut tout autant admettre que la logique classique est obtenue par la suppression de certaines règles logiques.

$\mathrm{A} \vdash \mathrm{A} \vee \mathrm{B}$ est une expression homophone et homographe appartenant aux idiomes classiques et intuitionistes et pour laquelle les locuteurs de ces deux langages donnent leur assentiment en toute circonstance. Le principe de l'indétermination de la traduction est ainsi établi sur les bases fermes et neutres de la logique mathématique. Du point de vue du langage de la logique classique, les constantes logiques intuitionistes peuvent paraître avoir " une sorte de sens intuitif " qui se rapproche "par une analogie grossière " des constantes de la logique classique, comme le dit Quine. Mais seulement au prix d'une appréciation encore plus grossière, qui voit ces constantes comme des isolats, sans considération pour la nature des constituants linguistiques auxquels elles sont inévitablement liées, nature qui est analysée par les règles de données du calcul des séquents. C'est uniquement en s'attachant à une vision archaïque de la logique, comparable à la conception euclidienne de la géométrie, qui mêle dans ses axiomes contenu intuitif et procédé déductif, qu'on peut privilégier une espèce de sens révélé aux constantes logiques indépendamment de la nature globale du langage de la logique. Les éléments de ce langage ne peuvent et ne doivent être compris qu'à travers l'examen des relations entretenues par ses différents constituants.

Nous pouvons passer à l'examen de notre problème qui est de savoir si la logique mathématique permet de concevoir un système logique possédant les règles de la logique classique et dont les données puissent désigner des énoncés

\section{Confusion et obscurité de la notion d'énoncé.}

Être philosophiquement neutre, ce n'est pas ignorer les engagements ontologiques, tels que les expriment partiellement mais distinctement les règles de données, auxquels nous astreignent les systèmes logiques, mais, au contraire, admettre que ceux-ci sont inévitables et qu'il faut les reconnaître pour en mesurer correctement la portée. Par conséquent, si les objets du calcul des prédicats sont des énoncés, on ne peut, du point de vue de la logique, en conclure qu'une chose : la neutralité ontologique de la logique signifie simplement que son ontologie est différente de celle de la théorie des ensembles. Mais la légitimité de cette distinction ne peut être reconnue que par un seul

23. Anderson, Alan et Belnap, Nuel, Entailment, Princeton, Princeton University Press, 1975. Les qualificatifs « additive » et «multiplicative » sont dûs à Girard, Jean-Yves, «Linear logic » Theoretical Computer Science 50, 1-102, 1987. 


\section{Philosophiques / Automne 2000}

tribunal : les règles de la logique. Celles-ci sont-elles sensibles à une telle distinction ? Et par quelles marques?

\subsection{Confusion.}

Bien que les objections de Quine à l'encontre des propositions ne proviennent pas «d'un refus des entités intangibles ou abstraites " 24 il n'en est pas moins vrai que la matérialité est une propriété qui oppose les énoncés aux propositions : des phénomènes physiques (ondes sonores ou lumineuses) signalent la présence des premiers et aucun signal physique ne nous indique la présence des secondes. ${ }^{25}$

Puisque, pour Quine, la logique, c'est le calcul classique des prédicats, il suffirait de vérifier que les règles de données par lesquelles le calcul des séquents distingue ce système logique (permutation, contraction, expansion et affaiblissement, chacune à gauche et à droite) sont satisfaites par les énoncés et ne le sont pas par les propositions pour que soit établie de façon drastique la thèse selon laquelle les objets de la logique classique sont des énoncés.

"Si nous avons chacun un oeuf, dit un proverbe chinois, et que nous l'échangeons, nous aurons chacun un oeuf. Si nous avons chacun une idée et que nous l'échangeons, nous aurons chacun deux idées. » Les propositions ressemblent aux idées. Les énoncés, dont on nous dit qu'ils se distinguent des propositions par leur matérialité, doivent-ils être mis dans le même panier que les œufs?

Avec les règles d'expansion et de contraction, le calcul des séquents dispose des ressources symboliques permettant d'exprimer l'opposition entre matérialité et immatérialité des données. Ces règles formalisent, comme l'a remarqué Girard, la nature suprasensible des objets de la logique classique ${ }^{26}$. Employons A dans une inférence d'une démonstration logique, nous pour-

24. Quine, Philosophy of Logic, p.3. Ici, comme l'a remarqué un des examinateurs de cet article, la traduction française de l'ouvrage de Quine doit être modifiée. Celle-ci dit : "entités non sensibles ou abstraits".

25. On préjuge peut-être de la nature des formes linguistiques lorsqu'on affirme qu'elles sont concrètes. Les énoncés sont des suites de phonèmes et un phonème n'a pas plus de réalité physique qu'une proposition. L'invocation (Philosophie de la logique, p.26) de la distinction peircéenne entre tokens (signes concrets) et type d'un énoncé pour caractériser les énoncés éternels, plutôt que de dissiper cette difficulté en ajoute une nouvelle. Puisqu'un phonème est une classe de sons équivalents (les membres d'une même classe d'équivalence pouvant être matériellement plus dissemblables entre eux que ne le sont des représentants de phonèmes distincts) et qu'un type est la classe des tokens équivalents, le caractère concret des énoncés paraît mal établi.

26. Girard, "Linear logic », art. cité. Cette distinction entre nature matérielle et nature immatérielle des données désignées par les formules de la logique doit être prise en compte lorsqu'on désire qu'un ordinateur fasse de la déduction logique puisque celui-ci, évidemment, ne manipule pas des abstraits, mais des chaînes de caractères qui sont bel et bien concrètes. Les règles d'expansion et de contraction du calcul des séquents permettent de traduire la distinction entre données matérielles et immatérielles de la façon suivante. Lorsque A apparaît dans un 
rons toujours la réemployer dans une inférence ultérieure. Employons un oeuf pour faire une pâte à biscuit, nous ne pourrons plus l'utiliser pour faire la crème pâtissière. La matérialité n'est pas un trait distinctif des objets du calcul classique des prédicats. Ceux-ci doivent être considérés comme des abstraits. La notion d'énoncé est donc confuse puisqu'elle est indistinguible de celle, abstraite, de proposition, qui, par cette seule abstraction, répond aux propriétés que le calcul des séquents assigne aux données de la logique classique. Des propriétés formelles d'une représentation neutre de la logique on ne peut conclure que les formules de la logique classique désignent des énoncés, celles-ci nous invitant plutôt à constater que les données de cette logique vérifient la propriété qui distingue les propositions des énoncés.

Le formalisme de la logique ne pouvant, à lui seul, épuiser les questions que l'on peut, et que l'on doit, se poser dans l'examen des rapports de la logique mathématique et de la théorie de la connaissance, on pourrait songer à objecter aux considérations précédentes qu'elles n'ont pas la valeur d'un test définitif. Mais jusqu'ici, on s'est astreint à respecter les principes de la dialectique, qui nous enjoignent d'accepter par provision les hypothèses et les méthodes reçues par ceux dont nous voulons rejeter les conclusions. Or Quine « appartient à la petite majorité de ceux qui rejettent le rêve cartésien d'un fondement de la méthode scientifique plus ferme que la méthode scientifique elle-même $"{ }^{27}$ Comme on ne peut concevoir de science plus ferme, c'est-à-dire plus neutre, que le calcul des séquents, qui n'est rien d'autre qu'une théorie symbolique générale de la représentation du raisonnement correct, il faut alors convenir que le test proposé à l'instant est à préférer, par exemple, aux arguments ressortant d'une théorie comportementaliste du langage qui, comme on le sait, ne reçoit pas l'assentiment de tous les linguis-

séquent, alors $\mathrm{A}, \mathrm{A}$ ou $\mathrm{A}, \mathrm{A}, \mathrm{A}$ ou $\mathrm{A}, \mathrm{A}, \mathrm{A}, \mathrm{A}$, ou etc... peuvent en être inférées (expansion). Inversement de A, A, A, A , par application réitérée de la contraction, A (seule) peut être inférée. C'est là une propriété distinctive des données immatérielles. Supprimons les règles d'expansion et de contraction : le calcul logique distinguera alors une occurrence singulière de A d'une double occurrence $\mathrm{A}, \mathrm{A}$. Les formules démontrables à partir d'un séquent, selon qu'il contient une ou deux occurrences de A, ne seront plus les mêmes.

Cette propriété a trouvé une part de sa signification à l'occasion des développements de l'application de la logique aux langages de programmation. Les ordinateurs, contrairement aux hommes, ne peuvent percevoir des êtres immatériels tels que les propositions. Leur comportement n'est affecté que par le caractère concret des symboles présents dans les formules logiques. Par conséquent l'information associée à deux nombres distincts d'occurrences d'un symbole donné ne peut être la même pour chacun de ces cas.

27. Quine, William V.O. Pursuit of Truth, Harvard, Harvard University Press, 1990, p.19. Cette majorité est-elle si étroite ? Tout laisse plutôt penser qu'elle est pratiquement absolue. Elle réunit, aux côtés de Quine, Wittgenstein, Heidegger et Foucault. La majorité a-telle raison ? Ce serait alors un cas exceptionnel dans l'histoire de la pensée philosophicoscientifique. Mais on doit tout autant convenir que si cette majorité est aussi large c'est au bénéfice de l'obscurité de la locution "méthode scientifique ". Ce que Quine, d'ailleurs, semble admettre par implication. Voyez par exemple «Success and limits of mathematization » in Quine, William V.O.,Theories and Things, Harvard University Press, 1981, pp.148-54. 
tes ou de tous les psychologues, et qui, par là même ne peut prétendre à la même neutralité que la logique mathématique.

\subsection{Obscurité.}

Derechef « Le propre des énoncés — d'où vient leur différence avec les autres formes linguistiques - est qu'ils sont susceptibles de vérité et de fausseté et peuvent donc être affirmés et niés de manière significative. »

Une forme linguistique peut-elle être définie par un critère non linguistique, tel que la vérité ? En d'autres termes la vérité délimite-t-elle, au sein de la classe des expressions linguistiques admissibles dans une langue naturelle donnée, une sous-classe distinguée dont on puisse dire que ses éléments sont eux-mêmes des formes linguistiques ?

Pour Quine les énoncés, au sens défini ci-dessus, sont des êtres linguistiques, car ils peuvent être caractérisés par un procédé propre à la définition des langages : la grammaire.

L'existence d'une grammaire pour le langage du calcul des prédicats est bien une condition suffisante pour affirmer que les expressions grammaticales de ce langage sont des expressions linguistiques. Toutefois la forme, sinon l'existence, d'une telle grammaire ne va pas de soi, même si l'exposé de Quine laisse penser le contraire. L'autorité de Quine en ces matières explique-t-elle le désintérêt des commentateurs, pourtant fort nombreux, pour cette question ? Quoi qu'il en soit, quand la critique suspend son assentiment à cet argument d'autorité elle se trouve face à des difficultés inextricables qui tiennent à l'origine confuse de la charge de la preuve. Il faut en effet trouver dans quelque chose, dont on nous dit qu'elle existe (une grammaire pour le langage du calcul des prédicats), mais dont on ne nous décrit pas la nature, l'inévitable défaut de construction qu'elle comprendrait si, au lieu de se contenter d'affirmer son existence, on s'avançait jusqu'à la décrire.

On conduira l'analyse de cette question comme suit. (1) On vérifiera d'abord, dans le cas simplifié du calcul des propositions, que les noms de propositions sont à la fois construits par la grammaire et donnés comme déjà construits. (2) On constatera ensuite que, dans toute grammaire pour le calcul des prédicats, ce procédé doit être multiplié pour les noms des prédicats, des variables et des individus. Comme le propos de Quine invite à confondre des matières que notre science permet de distinguer clairement, $(0)$ on fera précéder ces remarques de rappels élémentaires sur la notion de grammaire formelle.

Les observations précédentes ne concerneront que l'affirmation : « le langage du calcul des prédicats a une grammaire ». Mais Quine demande plus à la grammaire, de la logique. Celle-ci doit traiter les noms, qu'il s'agisse des noms communs ou des noms propres, comme des dispositifs notationnels redondants. Cette exigence n'est pas destinée à alléger la rédaction de la grammaire mais à sauver le slogan « être c'est être la valeur d'une variable ". $\mathrm{Si}$, par exemple, il y avait réellement des noms propres dans la grammaire, 
alors être, ce serait être la valeur d'une variable ou être un individu. C'est pourquoi (3) on essayera de faire voir que les procédés proposés pour éliminer les noms propres sont antinomiques avec l'idée de grammaire.

\section{(o) Rappels sur la grammaire}

Une grammaire est un quadruple $\left(\mathrm{S}, \mathrm{V}_{\mathrm{A}}, \mathrm{V}_{\mathrm{T}}, \mathrm{P}\right)$ où $\mathrm{S}$ est un axiome, $\mathrm{V}_{\mathrm{A}}$ et $\mathrm{V}_{\mathrm{T}}$ sont des vocabulaires distincts et finis, appelés respectivement vocabulaire auxiliaire et vocabulaire terminal, et $\mathrm{P}$ est un ensemble fini de règles de réécriture qui transforment des suites finies de symboles de $V_{A} \approx V_{T}$ en suites finies de symboles de $V_{A} \approx V_{T}$. L'ensemble des suites de symboles composées exclusivement d'éléments de $\mathrm{V}_{\mathrm{T}}$ engendrées par les règles de réécriture est le langage décrit par la grammaire. ${ }^{28}$

La distinction, dans une grammaire, entre vocabulaire auxiliaire et vocabulaire terminal correspond au fait que les éléments du vocabulaire auxiliaire doivent être réécrits, ils sont les analogues des parties du discours, tandis que les éléments du vocabulaire terminal ne peuvent être réécrits, ils correspondent aux mots du dictionnaire. C'est grâce à cette distinction qu'on peut considérer que le concept général de langage subsume la notion particulière de langage naturel ${ }^{29}$.

Cette description grammaticale des langages peut être dite purement syntaxique, car elle se ramène uniquement à des opérations de substitution de symboles. On remplace un ou plusieurs symboles par un ou plusieurs symboles (éventuellement par un symbole "nul », cas où la règle efface des symboles). Il faut, à ce sujet, rappeler une observation de $\mathrm{Chomsky}^{30}$. On appelle langage universel sur un vocabulaire donné l'ensemble de toutes les expressions que l'on peut construire à partir de ce vocabulaire. Cet ensemble est infini dénombrable. Par définition, un langage formel, sur ce vocabulaire, est un sous-ensemble du langage universel. L'ensemble des langages est donc non dénombrable. Comme une grammaire, par définition, ne comporte qu'un nombre fini de symboles, l'ensemble des grammaires est infini dénombrable. Il y a donc un ensemble non dénombrable de langages pour lesquels il ne peut exister de grammaire. En d'autres termes, avoir une grammaire, pour un langage, au sens large mais précis de la théorie des grammaires, est l'exception plutôt que la règle. Venons-en à la syntaxe logique.

28. Exemple: La grammaire $G=\left(S, V_{A}=\{A, B\}, V_{T}=\{a, b\}, P=\{S \rightarrow A S B, S \rightarrow A B\right.$, $A \rightarrow a, B \rightarrow b\})$ engendre le langage $L(G)=\left\{a^{n} b^{n}\right\}$ composé des expressions $a b$, aabb, aaabbb,...Le symbole $\rightarrow$ se lit «se réécrit » (on ne doit pas le confondre avec le symbole de l'implication matérielle $: \rightarrow)$. G engendre, par exemple, l'expression $\mathrm{a}^{2} \mathrm{~b}^{2}$ avec la dérivation suivante $\mathrm{S} \rightarrow \mathrm{ASB} \rightarrow \mathrm{AABB} \rightarrow \mathrm{aABB} \rightarrow \mathrm{aaBB} \rightarrow \mathrm{aabB} \rightarrow$ aabb en appliquant succesivement la première, la deuxième, la troisième, la troisième, la quatrième et la quatrième règle.

29. Cette observation est indépendante de la question de savoir s'il est possible de construire des grammaires, au sens retenu ici, pour les langages naturels.

30. Chomsky, Noam, «Trois modèles de la description du langage ", Langages $n^{\circ} 9$, 1968, pp.51-76. 
(1) Construction et emploi des noms des propositions dans toute grammaire pour le langage du calcul des propositions.

Commençons par simplifier l'exposé en ne retenant qu'un mot logique, $\left.\right|^{31}$, dans la rédaction des expressions du calcul des propositions.

Le langage le plus simple du plus simple des systèmes logiques est défini au moyen de

i) une liste indéfinie ${ }^{32}$ de variables propositionnelles : $\mathrm{p}_{1}, \mathrm{p}_{2}, \ldots$

ii) une constante logique : |

iii) règles de formation :

a) $\mathrm{p}_{1}, \mathrm{p}_{2}, \ldots$ sont des expressions bien formées

b) Si A et B sont des expressions bien formées alors (A $\mid$ B) est une expression bien formée.

L'application de ces règles permet d'obtenir l'ensemble des expressions bien formées du calcul classique des propositions. Chacune de ces expressions se présente comme un assemblage de symboles où ne figurent que des $\mathrm{p}_{\mathrm{i}}$, des | et des parenthèses, ces dernières indiquant l'ordre de la construction de l'expression et pouvant être omises au profit d'un autre dispositif notationnel tel qu'un indicateur syntagmatique ou arbre de construction. Une telle expression sera, par exemple $\left(\mathrm{p}_{\mathrm{i}} \mid \mathrm{p}_{\mathrm{j}}\right) \mid \mathrm{p}_{\mathrm{k}}$. On voit donc que, s'il faut assimiler l'ensemble des expressions du langage de la logique classique des propositions à un langage engendré par une grammaire, les $\mathrm{p}_{\mathrm{i}}$ doivent remplir le rôle, aux côtés de |, d'éléments du vocabulaire terminal. Or le fait que le nombre des $\mathrm{p}_{\mathrm{i}}$ soit indéfini, qu'il ne soit pas fini, est incompatible, par définition, avec un tel rôle. Ces éléments doivent eux-mêmes être engendrés par une règle de réécriture comme $\mathrm{p} \rightarrow \mathrm{p}$ '. Un artifice technique permet de contourner facilement cette difficulté. Prenons comme vocabulaire terminal $\mathrm{V}_{\mathrm{t}}=\{\mid, 1\}$ et comme règles de grammaire $\{\mathrm{S} \rightarrow \mathrm{S} \mid \mathrm{S}, \mathrm{S} \rightarrow \mathrm{p}, \mathrm{p} \rightarrow \mathrm{pp}, \mathrm{p} \rightarrow 1\}$, nous obtenons ainsi un procédé de renommage des variables propositionnelles qui satisfait les réquisits de la grammaire, c'est à dire qui permet de disposer d'un vocabulaire terminal dont les éléments ne sont pas réécrits.

(2) Duplication du procédé précédent pour engendrer le langage du calcul des prédicats du premier ordre.

On vient de voir que, pour pouvoir affirmer qu'un langage logique, même dans le plus simple des cas, a une grammaire, il faut recourir à un artifice technique. Cet artifice, traiter les éléments du vocabulaire terminal comme des éléments du vocabulaire auxiliaire, est inévitable, car la définition inducp.28.

31. |est la constante logique de Sheffer. Voir, par exemple, Quine, Méthodes de logique,

32. L'acception de "indéfini ", ici, est celle employée par Descartes : qui n'est ni fini ni infini ou, en d'autres termes, dont on ne peut connaître le nombre. 
tive des expressions bien formées du langage du calcul des propositions s'appuie sur la donnée d'un vocabulaire terminal dont on n'a pas à, et dont on ne peut, connaître le nombre des éléments. Cette observation vaut, à plus forte raison, pour le langage de la logique classique du premier ordre.

D'abord, comme le dit Quine ${ }^{33}$, les variables doivent être construites par une règle d'accentuation, $x \rightarrow \mathrm{x}$ '. Elle a le même effet que celle que nous venons d'examiner pour les noms de propositions. On peut donc la traiter de la même manière. Qu'en est-il des prédicats ? " Le logicien, dit Quine, se désintéresse d'expliciter complètement le lexique des prédicats puisqu'il est indifférent pour la structure du langage $»^{34}$. Mais la question présentement examinée n'est pas une question de logique, c'est une question de grammaire. Les définitions usuelles du langage du premier ordre stipulent qu'on a $\mathrm{P}_{1}{ }^{1}, \ldots, \mathrm{P}_{\mathrm{n}}{ }^{1}, \ldots$, $\mathrm{P}_{1}^{2}, \ldots, \mathrm{P}_{\mathrm{m}}{ }^{2}, \ldots, \mathrm{P}_{1}^{\mathrm{i}}, \ldots, \mathrm{P}_{1}^{\mathrm{i}}, \ldots$ prédicats dans le vocabulaire du langage, les exposants indiquant le nombre d'arguments qu'on doit lui juxtaposer pour obtenir une expression bien formée du langage, une prédication dans le langage de Quine. La différence entre le cas des noms de prédicats et celui des noms de variables ou des noms de propositions est dans le nombre de règles de réécriture qu'il faut employer pour les engendrer : il en faut deux fois plus. En fixant à l'avance la liste des prédicats, comme le demande Quine, en donnant son sens usuel et légitime à la notion de vocabulaire terminal, ou de lexique, on franchit le pas qui distingue le langage du calcul des prédicats du langage d'une théorie formulée dans le langage du calcul des prédicats. Aussi, soit on dit en toute rigueur que le langage du calcul des prédicats a une grammaire, mais alors c'est au prix d'une description formelle indifférenciée des éléments du vocabulaire auxiliaire et de ceux du vocabulaire terminal : ces éléments sont engendrés par des règles itératives qui ont pour effet commun de juxtaposer l'un à l'autre un même caractère. Soit il fallait entendre que la grammaire du langage de la logique, " dont la simplicité plaît " ${ }^{35}$, était la grammaire d'un emploi de ce langage (pour la formalisation d'une théorie).

Ces remarques suffisent à établir qu'il ne va pas de soi que le langage du calcul des prédicats a une grammaire.

\section{(3) Conséquences de l'élimination des noms.}

Il reste à considérer le dernier élément constitutif usuel du vocabulaire du calcul des prédicats, les constantes ou noms d'individus ou noms propres ${ }^{36}$.

Quand on retient pour acception de "grammaire du langage de la logique » celle de "grammaire d'un emploi du langage de la logique », on fixe la liste des prédicats qu'on peut alors ranger légitimement dans le vocabulaire terminal. Mais, implicitement, on fixe simultanément le nombre de

33. Quine, Philosophie de la logique, p.40.

34. Ibid., p.41.

35. Ibid., p.39

36. Pour simplifier l'exposé on néglige le cas des termes. 


\section{Philosophiques / Automne 2000}

noms de variables. Il est inutile d'avoir un nombre de ces noms supérieur à la somme de toutes les places d'arguments de tous les prédicats du langage de la théorie. Par contre le nombre de noms d'individus dans le langage d'une théorie du premier ordre peut-il être fixé ? L'emploi d'un prédicat tel que " être fils de » devrait alors être restreint arbitrairement. La présence, dans le vocabulaire d'un langage formel, d'une suite indéfinie de noms d'individus remet réellement en cause le caractère exclusif de la disjonction entre le vocabulaire auxiliaire et le vocabulaire terminal. Ce dernier fait implique qu'une grammaire, même conçue pour une théorie, pour un emploi du langage de la logique, tout en satisfaisant la lettre de la définition de la grammaire, en sacrifie l'esprit en abolissant la distinction, constitutive de la notion de grammaire, entre vocabulaire auxiliaire et vocabulaire terminal.

Pour cette raison, peut-être, mais plus vraisemblablement pour d'autres auxquelles nous avons fait allusion plus haut et sur lesquelles il n'y a pas lieu de s'étendre, Quine considère que les noms propres ne sont que des dispositifs redondants de la grammaire et propose de les éliminer en les transformant en prédicats. Il ne nous reste qu'à vérifier l'incompatibilité de ce dernier procédé avec la définition littérale de la grammaire.

"Jean mange » se traduit, dans le langage de la logique, par M(Jean). En définissant un nouveau prédicat J on peut éliminer "Jean ", M(Jean) se transcrivant alors $(\exists \mathrm{x}) \mathrm{J}(\mathrm{x}) \wedge \mathrm{M}(\mathrm{x})$. Pour conserver l'assurance d'unicité que nous donnait le nom il faut ajouter à cette transcription « de nouveaux énoncés ${ }^{37}$, tels que seul Jean puisse être vrai du prédicat J, à savoir :

$$
\text { Den : } \neg(\exists \mathrm{x})(\mathrm{Ey})(\mathrm{J}(\mathrm{x}) \wedge(\mathrm{J}(\mathrm{y}) \wedge \neg(\mathrm{x}=\mathrm{y})) .
$$

Mais pourquoi faut-il introduire de nouveaux énoncés ? La grammaire engendre toutes les expressions bien formées du langage et ces "nouveaux énoncés » sont bel et bien des expressions grammaticales. Il n’y a donc rien à introduire, la grammaire s'en charge toute seule. De plus Den est une condition logique qui, par elle-même, ne donne aucune indication sur la nature des restrictions grammaticales portant sur le nom de la variable qu'on pourra accoler à J. À vouloir nous faire confondre grammaire et logique on en est venu à les confondre réellement. Den est moins mal écrite comme suit :

$$
\neg\left(\exists \mathrm{x}_{\mathrm{i}}\right)\left(\exists \mathrm{x}_{\mathrm{j}}\right)\left(\mathrm{J}\left(\mathrm{x}_{\mathrm{i}}\right) \wedge \mathrm{J}\left(\mathrm{x}_{\mathrm{j}}\right) \wedge \neg\left(\mathrm{x}_{\mathrm{i}}=\mathrm{x}_{\mathrm{j}}\right)\right)
$$

$\mathrm{x}_{\mathrm{i}}$ et $\mathrm{x}_{\mathrm{j}}$ sont deux noms (de variables) dont la forme se distingue par le nombre de caractères identiques les composant. La condition logique Den impose des restrictions sur la suite de règles de réécriture Jean $\wedge \mathrm{J}(-) \wedge \mathrm{E}\left(\mathrm{x}_{\mathrm{i}}\right) \mathrm{J}\left(\mathrm{x}_{\mathrm{i}}\right)$ qui sont simplement celles-ci : si $\mathrm{x}_{\mathrm{i}}$ a été employé dans une règle précédente de la grammaire, alors il ne peut être accolé à J. On peut donc remarquer en passant que le procédé destiné à éliminer les noms revient simplement à changer un nom pour un autre : parmi les noms de variables, certains seront réservés 
exclusivement à certains prédicats. Il y aura toujours deux catégories de termes singuliers : les variables et les variables réservées aux noms. Les règles de la syntaxe seront simplement plus compliquées. Alors que dans "le système redondant ", on voyait clairement ce qui pouvait figurer dans un quantificateur, dans le système soi-disant non redondant, il faut formuler des restrictions contextuelles pour distinguer, parmi les noms de variables, ceux qui peuvent être utilisés comme le sont habituellement les noms de variable et ceux qui sont affectés à un prédicat unique et qui peuvent figurer dans le quantificateur liant la pseudo variable affectée à ce prédicat. Mais ce n'est pas la stérilité du procédé de dénominalisation qui nous intéresse ici.

La dénominalisation confisque, à chaque occurrence d'un nom propre, un nom de variables. La convention par laquelle on croyait pouvoir fixer le vocabulaire des variables ne vaut donc plus. Il faut, même dans le cas où on se limite à un emploi du langage du premier ordre, engendrer cette partie du vocabulaire terminal. En soi, ce fait ne change rien. Nous l'avons vu, il faut déjà disposer de ce procédé pour les noms d'individus. Par contre, chaque apparition d'un nouveau nom de variable, qu'il soit destiné à un emploi de variable ou de pseudo variable, implique l'apparition de nouvelles règles de grammaire destinées à stipuler les restrictions évoquées plus haut. En d'autres termes, la croissance du vocabulaire terminal entraîne celle des règles de grammaire. Et une grammaire dont les règles changent au cours de son emploi n'est plus une grammaire, car il n'y a pas d'entité sans identité.

Si ces observations sont exactes, alors la notion d'énoncé, produit éclectique de considérations logiques et grammaticales, est obscure.

\section{Conclusion}

Concluons par deux remarques.

S'interrogeant sur la question de savoir si la logique classique manipule des énoncés, on a choisi d'examiner la doctrine de Quine car, de tous les philosophes, c'est celui qui semble avoir défendu avec le plus de cohérence cette idée. On peut donc retenir par provision que la logique classique ne manipule pas des énoncés.

On ignorerait tout de la philosophie de Quine, si on songeait que, ne portant que sur la philosophie de la logique, les remarques exposées précédemment ne concernent qu'un aspect de cette philosophie. Le rôle central tenue par la logique dans cette théorie de la connaissance fait que, si la conception de la logique s'écroule, le reste s'écroule avec. Bien que cette étude ne concerne pas la philosophie de Quine, mais la nature des objets de la logique classique, elle a pour corollaire, si les raisonnements qui y sont exposés sont corrects, l'incohérence de cette théorie. ${ }^{38}$

38. De fructueuses discussions avec Gabriella Crocco et Bruno Gaume ont accompagné l'élaboration de cet article. L'auteur leur témoigne sa gratitude. Il remercie également Elisabeth Schwartz pour l'avoir encouragé, à l'issue d'une présentation orale, à publier ces remarques. 